Hodgkin-Lymphom

\title{
Chemotherapien im Vergleich
}

Die beiden Chemotherapieregimes ABVD8 und BEACOPP4+4 führen bei $\mathrm{Pa}$ tienten mit fortgeschrittenem HochrisikoHodgkin-Lymphom (Stadium III oder IV) $\mathrm{zu}$ einem vergleichbaren ereignisfreien (EFS) und Gesamtüberleben (OS). Da sich BEACOPP4+4 gegenüber ABVD8 weder als wirksamer noch als weniger toxisch erwies, sollte nach Ansicht von Patrice Carde und Kollegen vor der Auswahl eine sorgfältige Kosten-Nutzen-Analyse durchgeführt werden. Diesen Schluss ziehen die Forscher aus den Ergebnissen des EORTC 20012 Intergroup Trial.

549 Patienten erhielten in der PhaseIII-Studie entweder acht Zyklen Doxorubicin, Bleomycin, Vinblastin und Dacarbazin (ABVD8) oder vier Zyklen einer dosiseskalierten Therapie mit Bleomycin, Etoposid, Doxorubicin, Cyclophosphamid, Vincristin, Procarbazin und Prednison $\left(\mathrm{BEACOPP}_{\text {ekaliert }}\right)$ gefolgt von vier Zyklen BEACOPP baseline $_{\text {(BEACOPP4+4) }}$ ohne Strahlentherapie.

Das mediane Alter der Patienten lag bei 35 Jahren, $75 \%$ waren Männer, $74 \%$ hatten eine Stadium-IV-Erkrankung, $81 \%$ B-Symptome und 59\% einen Internatio- nal Prognostic Score (IPS) $\geq 4$. Das mediane Follow-up betrug 3,6 Jahre.

In beiden Armen wurde bei 82,5\% der Patienten ein komplettes oder ein nicht bestätigtes komplettes Ansprechen erreicht. Nach vier Jahren betrug die Rate für das EFS 63,7\% in der ABVD8-Gruppe versus $69,3 \%$ in der BEACOPP4+4-Gruppe (Hazard Ratio [HR] 0,86; $\mathrm{p}=0,312$ ), die Rate für das krankheitsfreie Überleben 85,8 versus $91,0 \%$ (HR 0,59; $\mathrm{p}=0,076)$ und das OS 86,7 versus $90,3 \%$ (HR 0,71; $\mathrm{p}=0,208$ ).

Sechs bzw. fünf Patienten starben an den Folgen von Nebenwirkungen, zwölf bzw. 26 Patienten brachen die Therapie vorzeitig ab.

Kathrin von Kieseritzky

Carde P et al. Eight Cycles of ABVD Versus Four Cycles of BEACOPPescalated Plus Four Cycles of BEACOPPbaseline in Stage III to IV, International Prognostic Score \$3, High-Risk Hodgkin Lymphoma: First Results of the Phase III EORTC 20012 Intergroup Trial. J Clin Oncol. 2016;34(17):2028-36.

\section{kurz notiert}

\section{Kolonkarzinom: einfacher Bluttest zur Abschätzung des Rezidivrisikos}

Wenn Krebszellen absterben, geben sie ihre DNA oftmals ins Blut ab. Durch neue Technologien ist es nun gelungen, diese zirkulierenden DNA-Fragmente zu analysieren. Dies nutzten Jeanne Tie und Kollegen in einer Untersuchung von 1.046 Blutproben von 230 Patienten und fanden heraus: Zirkulieren im Blut von Patienten mit Kolonkarzinom im Stadium II nach der Operation DNA-Fragmente, dann ist dies ein Zeichen dafür, dass der Tumor persistiert und ein hohes Rezidivrisiko besteht. Mit dem Test könnten Resterkrankungen in einem frühen Stadium erkannt werden, noch bevor dies mit klinischen oder radiologischen Kriterien möglich ist. Die Tests erfolgten mit Blutproben, die den Patienten vier bis zehn Wochen nach der Tumorresektion entnommen wurden. Bei 20 von 230 Patienten war zirkulierende DNA nachweisbar. 80 \% von ihnen entwickelten innerhalb von zwei Jahren ein Rezidiv. Dagegen war dies nur bei $10 \%$ der Patienten mit negativem Bluttest der Fall. Der Nachweis zirkulierender DNA-Fragemente liefert Hinweise darauf, dass sich noch Zellen des ursprünglichen Tumors im Körper befinden. Durch solche Bluttests könnte das Management von Patienten mit Kolonkarzinom erheblich verbessert werden [Tie J et al. Sci Transl Med. 2016;8(346):346ra92]. Judith Neumaier

\section{Chemotherapie bei Darmkrebs}

\section{Muskelschwund verkürzt das Leben}

Bei Patienten mit metastasiertem kolorektalem Karzinom ist Muskelschwund während der palliativen Chemotherapie mit einem verringerten Gesamtüberleben assoziiert. Bei mehr als 9\% Verlust an Muskeln leben die Patienten signifikant kürzer, wie aus den Ergebnissen einer prospektiven Studie hervorgeht.

Mithilfe computertomografischer Aufnahmen des Abdomens in Höhe der Lumbalregion (L3) wurde bei 67 Patienten die Skelettmuskelmasse vor Therapiebeginn und median nach 78 Tagen ermittelt. Das Follow-up lag bei 3,5 Jahren.

Im Mittel waren die Studienteilnehmer 66,4 $\pm 10,6$ Jahre alt, $63 \%$ waren Männer. Mit einem Anteil von $78 \%$ erhielten die meisten Patienten eine Erstlinienchemotherapie. $66 \%$ wurden mit einer Kombi- nation aus Capecitabin plus Oxaliplatin mit oder ohne Bevacizumab behandelt. $\mathrm{Zu}$ Studienbeginn waren $55 \%$ übergewichtig, $8 \%$ waren adipös. $57 \%$ hatten einen niedrigen Skelettmuskelindex, was

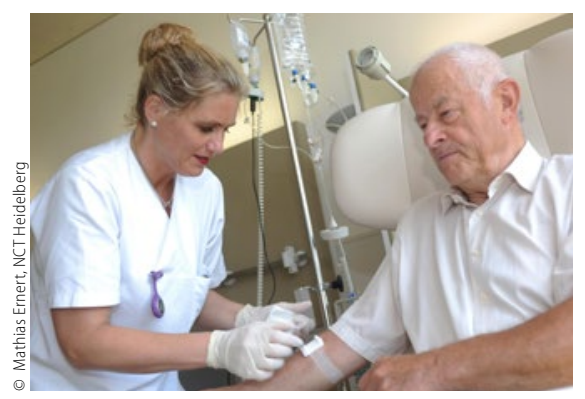

Wenn während der Chemotherapie die Muskeln schwinden, steigt das Sterberisiko. dem Quotienten aus Skelettmuskelfläche (Summe der Muskelquerschnitte auf Höhe L3) und Körperlänge entspricht.

Die Skelettmuskelfläche nahm bei beiden Geschlechtern innerhalb von drei Monaten unter der Chemotherapie um $6,1 \%(\mathrm{p}<0,001)$ ab. Median betrug das Gesamtüberleben bei Patienten mit Erstlinientherapie 17,5 Monate und bei Patienten mit einer Zweitlinientherapie 8,5 Monate.

Nach einem halben Jahr lebten noch $69 \%$ mit einem Muskelschwund < $9 \%$ versus $33 \%$ mit $\geq 9 \%$ Muskelschwund, nach einem Jahr noch 49 versus $17 \%$. Die Wahrscheinlichkeit zu sterben war für Patienten mit einem Muskelschwund über $9 \%$ gegenüber der Vergleichsgruppe um fast das 3,5-Fache erhöht. Peter Leiner

Blauwhoff-Buskermolen S et al. Loss of Muscle Mass During Chemotherapy Is Predictive for Poor Survival of Patients With Metastatic Colorectal Cancer. J Clin Oncol. 2016;34(12):1339-44. 